\title{
Agresión, victimización y autoestima en escolares con sobrepeso y obesidad
}

\author{
Aggression, victimization and self-esteem in \\ overweight and obesity school students
}

Eduardo Vilchis Chaparro*

\section{RESUMEN}

Objetivo: Evaluar la agresión, victimización, autoestima y tipología familiar en escolares con sobrepeso y obesidad. Material y métodos: Transversal, descriptivo. Criterios inclusión: Escolares de siete a 16 años con sobrepeso/obesidad, criterios exclusión: no firmen consentimiento informado. Tamaño de la muestra: 235 escolares, intervalo de confianza (IC): 90\%, proporción: 0.25, amplitud IC: 0.10. Variables: edad, sexo, IMC, actividad física semanal, violencia, victimización, agresión, autoestima, tipología familiar. Resultados: Los escolares proviene de familias nucleares simples en 50.70\%. Tipología por desarrollo: 41.4\% moderna, actividad física semanal: $43.8 \%$ no practican, $27.6 \%$ realizan una a dos horas por semana. Casi toda la población presentó algún grado de victimización, $70.6 \%$ sufrieron algún grado de agresión y violencia. Sexo femenino: $20.4 \%$ con baja autoestima, una cuarta parte que sufren sobrepeso y obesidad tiene baja autoestima. En la familia nuclear simple y tradicional en 36.1 y $28.9 \%$ sufrieron violencia respectivamente. Conclusiones: Padecer sobrepeso/obesidad tiene $29.3 \%$ la probabilidad de sufrir violencia. El sexo femenino tiende a ser agresora y víctima en una proporción 2:1. Más de la mitad de las víctimas, también ejercen algún grado de agresión y tienden en un futuro convertirse en agresores. Tres cuartas partes tenían un nivel medio de autoestima, la asociación entre ser víctima de violencia y ejercer agresión afecta su autoestima en sólo una cuarta parte de los escolares estudiados.

Palabras clave: Tipología familiar, sobrepeso, obesidad, violencia, agresión, victimización, autoestima.

\begin{abstract}
Objective: To assess aggression, victimization, self-esteem and family typology in school students who are overweight and obese. Material and methods: Transversal, descriptive. Inclusion criteria: school students aged 7-16 with overweight/obesity, exclusion criteria: do not sign informed consent. Sample size: 235 schoolchildren, confidence interval (Cl): 90\%, proportion: 0.25, amplitude $\mathrm{Cl}$ : 0.10. Variables: age, sex, BMI, weekly physical activity, violence, victimization, aggression, self-esteem, family typology. Results: School children come from simple nuclear families in 50.70\%. Typology by development: $41.4 \%$ modern, weekly physical activity: $43.8 \%$ do not practice, $27.6 \%$ do one to two hours per week. Almost the entire population presented some degree of victimization, $70.6 \%$ suffered some degree of aggression and violence. Female sex: $20.4 \%$ with low self-esteem, a quarter who are overweight and obese have low self-esteem. In the simple and traditional nuclear family, 36.1\% and $28.9 \%$ suffered violence, respectively. Conclusions: Being overweight/obese has a $29.3 \%$ probability of suffering violence. The female sex tends to be aggressor and victim in a 2:1 ratio. More than half of the victims also exert some degree of aggression and tend to become aggressors in the future. Three quarters had a medium level of selfesteem, the association between being a victim of violence and exercising aggression affects their self-esteem in only a quarter of the students studied.
\end{abstract}

Keywords: Family typology, overweight, obesity, violence, aggression, victimization, self-esteem.
* Médico Familiar. Maestro en Ciencias de la Educación, Doctor en Ciencias para la Familia, Doctor en Alta Dirección en Establecimientos de Salud. Centro de Investigación Educativa Formación Docente Siglo XXI, Centro Médico Nacional Siglo XXI, Instituto Mexicano del Seguro Social.

Correspondencia: EVC, lalovilchis@gmail.com Conflicto de intereses: El autor declara que no tiene. Citar como: Vilchis CE. Agresión, victimización y autoestima en escolares con sobrepeso y obesidad. Rev CONAMED. 2020; 25(2): 57-65. doi: $10.35366 / 94388$ Financiamiento: Para poder llevar a cabo esta investigación, se contó con un aplicador y recolector de datos (Eduardo Vilchis Chaparro). Se utilizó el cuestionario MBI, así como copias de éste. Se hizo uso de las instalaciones de Hospital General de Zona con Unidad de Medicina Familiar No. 8 «Dr. Gilberto Flores Izquierdo». Todos los gastos corrieron a cargo del investigador Eduardo Vilchis Chaparro.

Recibido: 13/05/2020. Aceptado: 15/06/2020. 


\section{INTRODUCCIÓN}

El intentar entender el motivo y razones de la conducta agresiva, obesidad y su influencia con la autoestima, ha dado pie a investigaciones en el tema, colocándola como una reacción a estímulos aversivos, como respuesta innata ante las situaciones peligrosas o incluso como una conducta intencionada. Sin embargo, algunos beneficios sociales de la agresión y la autoestima es el orden a partir de las jerarquizaciones o relaciones diferenciales; por lo tanto, mantener un balance adecuado entre la agresión y respeto tiene su complejidad en los contextos sociales, personales y culturales donde se desarrolla; de ahí la importancia de analizar estos aspectos de manera integral.'

Relacionado con lo anterior, define que una de las principales consecuencias de la obesidad (considerada la más importante) es la pérdida de la autoestima, la cual puede llevar a la persona a desencadenar un episodio depresivo, por lo que algunas personas tratan de compensar dicha afectación, con la ingesta excesiva de alimentos hipercalóricos para aliviarla. ${ }^{2}$

La violencia es una constante en cualquier ambiente escolar y familiar, no exclusivo de alguna clase social o en un sexo en específico, aunque el perfil del que infringe violencia (agresor) predomina en los varones. No existe diferencia en lo que respecta a los que sufren la agresión (víctima). El agresor acosa a la víctima cuando se encuentra solo. Por esta razón los maestros en muchas ocasiones no poseen información de la agresión. Lo que resulta en la formación de ambientes violentos que, si no se corrigen de forma temprana, puede propiciar severos daños físicos y emocionales a la víctima. ${ }^{3}$

Este es un fenómeno que normalmente ocurre entre dos (o más) iguales, con una igualdad relativa de edad. A pesar de esta coincidencia, siempre existe un desbalance entre los participantes (de poder) con el fin de que a través de la acción de abuso se domine e intimide al otro, a través de actos negativos deliberados, reiterados, persistentesy sistematizados. En el caso del bullying no siempre hay repercusiones físicas, pero inherentemente siempre existe daño psicológico y emocional. ${ }^{4}$

En la actualidad la participación femenina en el campo laboral es más amplia. Esto ha llevado a afectar su vida personal principalmente en el ámbito familiar que conduce a conductas nutricionales no saludables, disminución del tiempo para realizar actividad física y el reemplazado de estas en actividades sedentarias, favoreciendo un ambiente obesogénico, aunado al sentimiento de culpa por la falta de tiempo que está dedica al cumplimiento de funciones familiares básicas aunado a la abundancia de distracciones y alimentos con alto contenido calórico. ${ }^{5}$

Por lo tanto, el objetivo del estudio es evaluar el nivel de autoestima, tipología familiar, victimización, agresión y violencia en escolares con sobrepeso y obesidad que acudieron a consulta de una unidad médica de primer nivel de atención.

\section{MATERIAL Y MÉTODOS}

Estudio transversal, descriptivo. Criterios inclusión: escolares de siete a 16 años con sobrepeso/obesidad, criterios exclusión: no firmen consentimiento informado. Criterios eliminación: no derechohabientes, con enfermedades crónico degenerativas, cuestionarios incompletos. Muestra: 235 escolares, aleatoria, IC: 90\%, proporción: 0.25, amplitud IC: 0.10. Variables: edad, sexo, IMC, tipología familiar, actividad física semanal fuera de la escuela, participación de padres en actividad física semanal de hijos(as). Victimización, agresión y autoestima. Se aplicó la prueba de autoestima para niños y adolescentes (Caso \& Hernández-Guzmán), conformada por 21 reactivos con un alfa de Cronbach $=0.88$, agrupados en cuatro factores: cogniciones sobre sí mismo, cogniciones de competencia, relación familiar y enojo. ${ }^{6}$ Se aplicó escalas de agresión y victimización de Orpinas y Frankowski, con escalas de agresión (11 ítems) y de victimización (10 ítems), las cuales miden conductas de agresión física y verbal entre estudiantes; éstas respuestas son sumativas, puntaje mínimo es 0 y el máximo es 66, con un alfa de Cronbach = 0.87. Para la obtención de los resultados: 0: nula frecuencia ser agresor, 1-22: baja frecuencia, 23-44 puntos: mediana frecuencia. 45-66 puntos: alta frecuencia. ${ }^{7}$ El análisis estadístico se llevó a cabo a través del programa (SPSS V22). El diagnóstico de sobrepeso es el IMC para la edad con más de una desviación típica por encima de la mediana establecida en los patrones de crecimiento infantil de la OMS. La obesidad es 
mayor que dos desviaciones estándar por encima de la mediana establecida en los patrones de crecimiento infantil de la OMS. Para el análisis de los resultados se utilizaron medidas de tendencia central, dispersión, frecuencias y proporciones. El presente estudio fue autorizado por el comité ético institucional, y se requirió consentimiento informado firmado de los escolares y sus padres y/o tutores responsables.

\section{RESULTADOS}

Se estudiaron 235 escolares con sobrepeso y obesidad; 144 (61.3\%) femenino y 91 (38.7\%) masculino. Edad de presentación: mínimo siete años y máximo 16 años, media 9.98 años, desviación estándar de 2.25 años (Tabla 7).

Un apartado estudiado fue la autoestima y su relación con sexo donde: sexo femenino 48 (20.4\%) nivel bajo de autoestima, 96 (40.9\%) nivel medio de autoestima. Sexo masculino 12 (5.1\%) nivel bajo de autoestima, 75 (31.9\%) nivel medio de autoestima y 4 (1.7\%) nivel alto de autoestima (Tabla 2).

En relación a la clasificación de IMC (OMS) y nivel de autoestima: sobrepeso 32 (13.6\%) nivel bajo de autoestima, 78 (33.2\%) nivel medio de autoestima y 4 (1.7\%) nivel alto de autoestima. En obesidad 28 (11.9\%) nivel bajo de autoestima, 93 (39.6\%) nivel medio de autoestima (Tabla 3).

En la asociación de sufrir violencia y nivel de autoestima: sí sufrió violencia 38 (16.2\%) nivel bajo de autoestima, 127 (54\%) nivel medio de autoestima y 1 (0.4\%) nivel alto de autoestima; aquellos que no sufrieron violencia 22 (9.4\%) nivel bajo de autoestima, 44 (18.7\%) nivel medio de autoestima, finalmente 3 (1.3\%) nivel alto de autoestima (Tabla 4).

Frecuencia de victimización y el nivel de autoestima: nula victimización 7 (3\%) nivel medio de autoestima, $1(0.4 \%)$ nivel alto de autoestima. Frecuencia baja de victimización 28 (11.9\%) nivel bajo de autoestima, 91 (38.7\%) nivel medio de autoestima, 3 (1.3\%) nivel alto de autoestima (Tabla 5).

La asociación entre ejercer violencia y el nivel de autoestima: ejercieron violencia 10 (4.3\%) nivel bajo de autoestima, 39 (16.6\%) nivel medio de autoestima. Aquellos que no ejercen violencia: 50 (21.30\%) nivel bajo de autoestima, 132 (56.2\%) nivel medio de autoestima y 4 (1.7\%) nivel alto de autoestima (Tabla 6).
La relación entre frecuencia de ser agresor y nivel de autoestima: nula frecuencia de agresión 6 (2.6\%) nivel bajo de autoestima, 27 (11.5\%) nivel medio de autoestima. A los de mediana frecuencia de agresión 46 (19.6\%) nivel bajo de autoestima, 123 (53.3\%) nivel medio de autoestima, 4 (1.7\%) nivel alto es autoestima. Para los de baja frecuencia de agresión: 8 (3.4\%) nivel bajo de autoestima, 20 (8.5\%) nivel medio de autoestima (Tabla 7).

En el rubro ser agresor y sexo: para el sexo femenino 19 (8.1\%) frecuencia nula a ser agresor, 107 (45.5\%) frecuencia baja a ser agresor, 17 (7.2\%) frecuencia media a ser agresor, 1 (0.4\%) frecuencia alta a ser agresor. En el sexo masculino: 14 (6\%) frecuencia nula a ser agresor, 66 (28.1\%) frecuencia baja a ser agresor, 11 (4.7\%) frecuencia media a ser agresor (Tabla 8).

Mientras que para la frecuencia de victimización y sexo: sexo femenino 3 (1.3\%) frecuencia nula a victimización, 79 (33.6\%) frecuencia baja a victimización, 47 (20\%) frecuencia mediana a victimización, 15 (6.4\%) frecuencia alta a victimización. Mientras que en el sexo masculino: 5 (2.1\%) frecuencia nula a la victimización, 43 (18.3\%) frecuencia baja a la victimización, 40 (17\%) frecuencia mediana a la victimización y 3 (1.3\%) frecuencia alta a victimización (Tabla 9).

En el rubro de tipología familiar y sufrieron violencia encontramos: familia nuclear simple: 85 (36.1\%) sí sufrieron violencia, 34 (14.5\%) no sufrieron violencia. Familia nuclear numerosa: 5 (2.1\%) sí sufrieron violencia, 3 (1.3\%) no sufrieron violencia; familia nuclear ampliada: 9 (3.8\%) sí sufrieron violencia, 4 (1.7\%) no sufrieron violencia. En familia binuclear: 13 (5.5\%) sí sufrieron violencia, 7 (3\%) no sufrieron violencia; familia extensa: 30 (12.8\%) sí sufrieron violencia, 12 (5\%) no sufrieron violencia, familia monoparental: 24 (10.2\%) sí sufrieron violencia y 9 (3.8\%) no sufrieron violencia (Tabla 10).

En el rubro de tipología familiar (desarrollo) y sufrieron violencia encontramos: tradicional: 68 (28.9\%) sí sufrieron violencia, 29 (12.3\%) no sufrieron violencia. Moderna: 98 (41.7\%) sí sufrieron violencia, 40 (17\%) no sufrieron violencia. En el apartado de tipología familiar (desarrollo) y ejercieron violencia analizamos: tradicional: 77 (32.7\%) sí ejercieron violencia, 20 (8.5\%) no ejercieron violencia. Moderna: 109 (46.3\%) sí ejercieron violencia, 29 (12.3\%) no ejercieron violencia (Tabla 77). 


\begin{tabular}{|c|c|c|c|}
\hline Apartado / Tópico & n (\%) & Apartado / Tópico & n (\%) \\
\hline Edad (años) & & Hacen tres a cuatro horas & $43(18.20)$ \\
\hline $7-9$ & $117(49.80)$ & Practican cinco o más horas & $24(10.30)$ \\
\hline $10-12$ & $81(34.50)$ & Participación semanal de los padres & \\
\hline $13-15$ & $35(14.90)$ & en la actividad física de los hijos(as) & \\
\hline $16-18$ & $2(0.90)$ & Intervienen cero veces por semana & $160(68.00)$ \\
\hline Escolaridad & & Lo hacen una vez & $32(13.80)$ \\
\hline Primaria & $175(74.50)$ & Dos veces & $29(12.30)$ \\
\hline Secundaria & $54(23.00)$ & Lo ejercen tres o más veces & $14(5.90)$ \\
\hline Preparatoria & $6(2.60)$ & Edad a la que el tutor notó el inicio de & \\
\hline El sexo del familiar que lo llevó a & & sobrepeso y obesidad (años) & \\
\hline recibir atención & & Desconoce & $29(12.30)$ \\
\hline Femenino & $193(82.10)$ & Menor del año & $18(7.70)$ \\
\hline Masculino & $42(17.90)$ & $1-3$ & $38(16.20)$ \\
\hline Parentesco del paciente & & $4-6$ & $79(33.60)$ \\
\hline Madre & $182(77.40)$ & $7-9$ & $50(21.30)$ \\
\hline Padre & $43(18.30)$ & Mayor de 10 años & $21(8.90)$ \\
\hline Abuelo(a) & $8(3.40)$ & Sí sufrió violencia & \\
\hline Otro familiar & $2(0.90)$ & Sí la presentó & $166(70.60)$ \\
\hline La edad del familiar con el que acudió a & & No la sufrió & $69(29.40)$ \\
\hline la unidad & & Sí sufrieron violencia y quién fue el & \\
\hline 20-30 años & $71(30.20)$ & agresor & \\
\hline 31-40 años & $107(45.50)$ & Compañeros & $128(54.50)$ \\
\hline 41-50 años & $50(21.30)$ & Maestros & $10(4.30)$ \\
\hline Mayores de 51 años & $7(3.00)$ & Ambos & $27(11.90)$ \\
\hline Estado civil & & Sí ejerció violencia (es agresor) & \\
\hline Soltero & $36(15.30)$ & Sí la ejerció & $49(20.90)$ \\
\hline Casado & $103(43.80)$ & No la ejerció & $186(79.10)$ \\
\hline Divorciado & $27(11.50)$ & Índice de masa corporal (IMC) & \\
\hline Viudo & $4(1.70)$ & Sobrepeso & $114(48.50)$ \\
\hline Unión libre & $53(22.60)$ & Obesidad & $121(51.50)$ \\
\hline Separado & $12(5.10)$ & Nivel de autoestima & \\
\hline Tipología familiar por estructura & & Bajo & $60(25.50)$ \\
\hline Tipología nuclear simple & $119(50.63)$ & Medio & $171(72.80)$ \\
\hline Nuclear numerosa & $8(3.04)$ & Alto & $4(1.70)$ \\
\hline Estructura nuclear ampliada & $13(5.50)$ & Frecuencia de agresión & \\
\hline Estructura binuclear & $20(8.50)$ & Nula & $33(14.00)$ \\
\hline Estructura extensa & 42 (17.87) & Baja & $173(73.60)$ \\
\hline Tipología monoparental & $33(14.04)$ & Mediana & $28(11.90)$ \\
\hline Tipología familiar por desarrollo & & Alta & $1(0.40)$ \\
\hline Familias modernas & $97(41.27)$ & Frecuencia de victimización & \\
\hline Familias tradicionales & 138 (58.72) & Nula & $8(3.40)$ \\
\hline Actividad física semanal fuera de la & & Baja & $122(51.90)$ \\
\hline escuela & & Mediana & $87(37.00)$ \\
\hline No la practican & $103(43.80)$ & Alta & $18(7.70)$ \\
\hline Realizan una a dos horas & $65(27.60)$ & & \\
\hline
\end{tabular}


Tabla 2: Sexo.

\begin{tabular}{|c|c|c|c|c|c|c|c|c|}
\hline & \multicolumn{8}{|c|}{ Nivel de autoestima } \\
\hline & \multicolumn{2}{|c|}{ Bajo } & \multicolumn{2}{|c|}{ Medio } & \multicolumn{2}{|c|}{ Alto } & \multicolumn{2}{|c|}{ TOTAL } \\
\hline & Frec. & $\%$ & Frec. & $\%$ & Frec. & $\%$ & Frec. & $\%$ \\
\hline Femenino & 48 & 20.4 & 96 & 40.9 & 0 & 0.0 & 144 & 61.0 \\
\hline Masculino & 12 & 5.1 & 75 & 31.9 & 4 & 1.7 & 91 & 38.7 \\
\hline TOTAL & 60 & 25.5 & 171 & 72.8 & 4 & 1.7 & 235 & 100.0 \\
\hline
\end{tabular}

Tabla 3: Sobrepeso y obesidad según IMC (OMS).

\begin{tabular}{|c|c|c|c|c|c|c|c|c|}
\hline & \multicolumn{8}{|c|}{ Nivel de autoestima } \\
\hline & \multicolumn{2}{|c|}{ Bajo } & \multicolumn{2}{|c|}{ Medio } & \multicolumn{2}{|c|}{ Alto } & \multicolumn{2}{|c|}{ TOTAL } \\
\hline & Frec. & $\%$ & Frec. & $\%$ & Frec. & $\%$ & Frec. & $\%$ \\
\hline Sobrepeso & 32 & 13.6 & 78 & 33.2 & 4 & 1.7 & 114 & 48.5 \\
\hline Obesidad & 28 & 11.9 & 93 & 39.6 & 0 & 0.0 & 121 & 51.5 \\
\hline TOTAL & 60 & 25.5 & 171 & 72.8 & 4 & 1.7 & 235 & 100.0 \\
\hline
\end{tabular}

Tabla 4: Sufrió violencia.

\begin{tabular}{|c|c|c|c|c|c|c|c|c|}
\hline & \multicolumn{8}{|c|}{ Nivel de autoestima } \\
\hline & \multicolumn{2}{|c|}{ Bajo } & \multicolumn{2}{|c|}{ Medio } & \multicolumn{2}{|c|}{ Alto } & \multicolumn{2}{|c|}{ TOTAL } \\
\hline & Frec. & $\%$ & Frec. & $\%$ & Frec. & $\%$ & Frec. & $\%$ \\
\hline Sí & 38 & 16.2 & 127 & 54.0 & 1 & 0.4 & 166 & 70.6 \\
\hline No & 22 & 9.4 & 44 & 18.7 & 3 & 1.3 & 69 & 29.4 \\
\hline TOTAL & 60 & 25.5 & 171 & 72.8 & 4 & 1.7 & 235 & 100.0 \\
\hline
\end{tabular}

\section{DISCUSIÓN}

La violencia es un fenómeno que siempre ha existido en nuestra sociedad, sin embargo, en los últimos tiempos el grado de violencia es tal que incluso ha costado la vida a quienes la sufren. Encontramos que aumenta la presencia de violencia en casi una tercera parte por el hecho de tener sobrepeso y obesidad.
En esta investigación se encontró de acuerdo a la escala de agresión y victimización adaptadas al español en casi la totalidad de la población escolar presentó algún grado de victimización, siendo en su mayoría con un 51.9\% a una frecuencia baja en victimización y más de dos terceras partes presenta algún grado de agresión, siendo el 73.60\% con frecuencia baja a la agresión. Mientras que la 
prueba de autoestima para niños y adolescentes una cuarta parte de la población estudiada tiene un nivel bajo de autoestima y $72.8 \%$ para nivel medio de autoestima.

Los programas deben estar más enfatizados en el sexo femenino, ya que más de la mitad de ellas son más propensas a sufrir violencia con una proporción 2:1 en comparación con el sexo masculino. De la misma forma las escolares de género femenino tienden a presentar más conductas agresivas en comparación con los del género masculino (2:1). De tal forma que se puede decir que las escolares del sexo femenino tienen más riesgo para ser víctima y también para ser agresoras.

Otra manifestación hallada es como las víctimas de violencia, también ejercen algún grado de agresión a sus compañeros como forma de defensa a sus ataques, si bien encontramos que más de la mitad de las víctimas, también ejercen algún grado de agresión a sus iguales. Esto es de suma importancia ya que los especialistas señalan que hasta uno de cada diez; este tipo de víctimas tienden en un futuro a corto-mediano plazo convertirse en agresores y así, la violencia en la escuela es un círculo vicioso que nunca termina.

Por otra parte, como ya se mencionó la mayoría de los escolares contaban con una autoestima media, es relevante notar que ningún escolar obeso tenía un nivel de autoestima alto; esto puede deberse a que los trastornos de la imagen corporal, salen del estándar de «belleza» actual, siendo esto un impedimento para que se eleve su nivel de autoestima.

Es sabido que la autoestima está formada por múltiples rubros que integran a un individuo. En este aspecto podemos enfatizar que el hecho de padecer sobrepeso y obesidad no marca del

Tabla 5: Frecuencia de victimización.

Nivel de autoestima

\begin{tabular}{|c|c|c|c|c|c|c|c|c|}
\hline & \multicolumn{2}{|c|}{ Bajo } & \multicolumn{2}{|c|}{ Medio } & \multicolumn{2}{|c|}{ Alto } & \multicolumn{2}{|c|}{ TOTAL } \\
\hline & Frec. & $\%$ & Frec. & $\%$ & Frec. & $\%$ & Frec. & $\%$ \\
\hline Nula & 0 & 0.0 & 7 & 3.0 & 1 & 0.4 & 8 & 3.4 \\
\hline Baja & 28 & 11.9 & 91 & 38.7 & 3 & 1.3 & 122 & 51.9 \\
\hline Mediana & 27 & 11.5 & 60 & 25.5 & 0 & 0.0 & 87 & 37.0 \\
\hline Alta & 5 & 2.1 & 13 & 5.5 & 0 & 0.0 & 18 & 7.7 \\
\hline TOTAL & 60 & 25.5 & 171 & 72.8 & 4 & 1.7 & 235 & 100.0 \\
\hline
\end{tabular}

Tabla 6: Ejerció violencia.

Nivel de autoestima

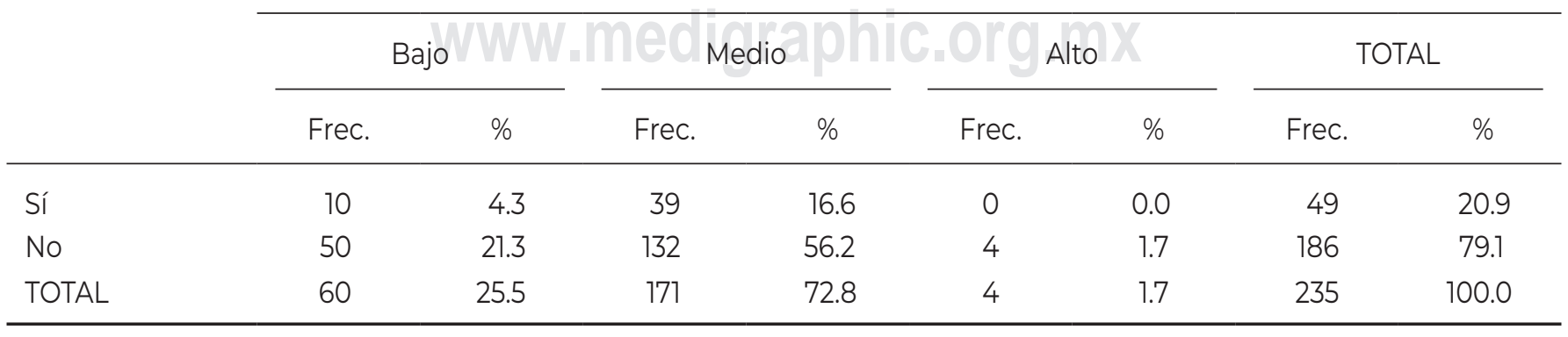


Tabla 7: Frecuencia de agresión.

\begin{tabular}{|c|c|c|c|c|c|c|c|c|}
\hline & \multicolumn{8}{|c|}{ Nivel de autoestima } \\
\hline & \multicolumn{2}{|c|}{ Bajo } & \multicolumn{2}{|c|}{ Medio } & \multicolumn{2}{|c|}{ Alto } & \multicolumn{2}{|c|}{ TOTAL } \\
\hline & Frec. & $\%$ & Frec. & $\%$ & Frec. & $\%$ & Frec. & $\%$ \\
\hline Nula & 6 & 2.6 & 27 & 11.5 & 0 & 0.0 & 33 & 14.0 \\
\hline Baja & 46 & 19.6 & 123 & 52.3 & 4 & 1.7 & 173 & 73.6 \\
\hline Mediana & 8 & 3.4 & 20 & 8.5 & 0 & 0.0 & 28 & 11.9 \\
\hline Alta & 0 & 0.0 & 1 & 0.4 & 0 & 0.0 & 1 & 0.4 \\
\hline TOTAL & 60 & 25.5 & 171 & 72.8 & 0 & 0.0 & 235 & 100.0 \\
\hline
\end{tabular}

Tabla 8: Frecuencia de ser agresor.

\begin{tabular}{|c|c|c|c|c|c|c|c|c|c|c|}
\hline \multirow[b]{2}{*}{ Sexo } & \multicolumn{2}{|c|}{ Nula } & \multicolumn{2}{|c|}{ Baja } & \multicolumn{2}{|c|}{ Mediana } & \multicolumn{2}{|c|}{ Alta } & \multicolumn{2}{|c|}{ TOTAL } \\
\hline & Frec. & $\%$ & Frec. & $\%$ & Frec. & $\%$ & Frec. & $\%$ & Frec. & $\%$ \\
\hline Femenino & 19 & 8.1 & 107 & 45.5 & 17 & 7.2 & 1 & 0.4 & 144 & 61.3 \\
\hline Masculino & 14 & 6.0 & 66 & 28.1 & 11 & 4.7 & 0 & 0.0 & 91 & 38.7 \\
\hline TOTAL & 33 & 14.0 & 173 & 73.6 & 28 & 11.9 & 1 & 0.4 & 235 & 100.0 \\
\hline
\end{tabular}

\begin{tabular}{|c|c|c|c|c|c|c|c|c|c|c|}
\hline \multirow[b]{2}{*}{ Sexo } & \multicolumn{2}{|c|}{ Nula } & \multicolumn{2}{|c|}{ Baja } & \multicolumn{2}{|c|}{ Mediana } & \multicolumn{2}{|c|}{ Alta } & \multicolumn{2}{|c|}{ TOTAL } \\
\hline & Frec. & $\%$ & Frec. & $\%$ & Frec. & $\%$ & Frec. & $\%$ & Frec. & $\%$ \\
\hline Femenino & 3 & 1.3 & 79 & 33.6 & 47 & 20 & 15 & 6.4 & 144 & 61.3 \\
\hline Masculino & 5 & 2.1 & 43 & 18.3 & 40 & 17 & 3 & 1.3 & 91 & 38.7 \\
\hline TOTAL & 8 & 3.4 & 122 & 51.9 & 87 & 37 & 18 & 7.7 & 235 & 100.0 \\
\hline
\end{tabular}

todo el nivel de autoestima en un escolar, ya que tres cuartas partes de ellos tenían un nivel medio de autoestima; así también la asociación entre ser víctima de violencia y ejercer agresión afecta su autoestima en sólo una cuarta parte de la muestra estudiada. Con esto se puede señalar que la autoestima la conforman otros componentes dentro del individuo y no solo ser obeso o sufrir de violencia, ya que puede estar determinada por la dinámica familiar, su tipología y otros factores.

\section{CONCLUSIONES}

Después de revisar los resultados de la presente investigación, surge la necesidad de diseñar acciones preventivas que permitan frenar la aparición de casos de violencia. Un programa de prevención la violencia que involucre a docentes y padres de familia, para evitar el maltrato entre iguales por medio de la sensibilización grupal, inculcando a los alumnos actitudes, valores y normas de convivencia positivas y fomentando 
relaciones interpersonales cordiales y mutuamente satisfactorias.

La finalidad es prevenir la violencia por medio de la sensibilización sobre el tema, para lo cual es importante: adquirir conciencia de la importancia de mantener relaciones interpersonales cordiales, positivas y satisfactorias con los iguales; aprender a reconocer, evitar y controlar las situaciones de riesgo o de maltrato que puedan darse, así como lograr despertar una conciencia en los niños y jóvenes de que tienen que informar y dar a conocer las situaciones de abuso que les sucedan y/o las que observen; desarrollar habilidades personales de autoconocimiento y autorregulación emocional y conductual, potenciando a su vez mecanismos de autoprotección y seguridad personal; adquirir habilidades de interacción social, de solución de problemas, de petición de ayuda y de asertividad; finalmente, se persigue lograr una implicación activa de padres, hijos y docentes en una cultura de no violencia.

El papel del médico de familia es siempre enfatizar el enfoque de riesgo, tanto en la prevención como el seguimiento de los pacientes que ya cuentan con alguno. Una de las visiones es siempre enfatizar un ambiente de confianza para facilitar y fomentar la comunicación entre el paciente y los miembros de la familia, de tal forma poder fomentar redes de apoyo y dar pautas que procuren el aumento de la autoestima y así, la disminución y erradicación de víctimas de violencia. El tratamiento debe ser integral para facilitar no solo herramientas que le permitan un cambio de hábitos alimentarios y de estilo de vida, sino también debe proveer de herramientas básicas para

Tabla 10: Tipología familiar (estructura)

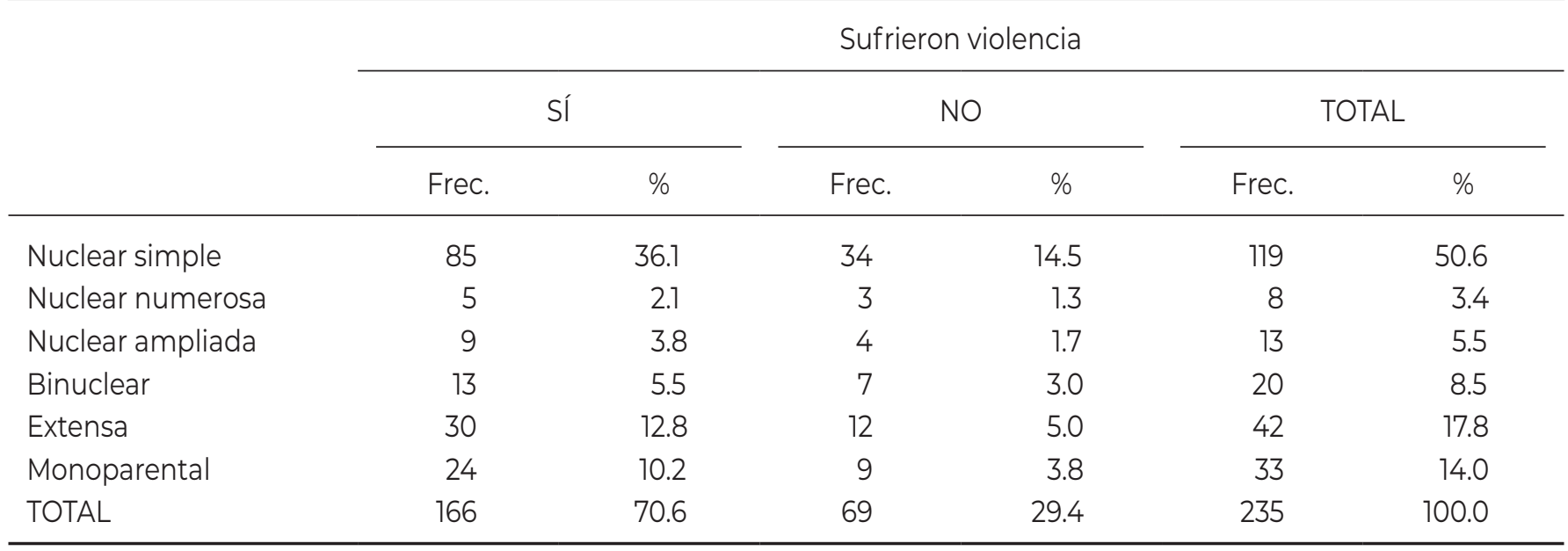

Tabla 11: Tipología familiar (desarrollo).

\begin{tabular}{|c|c|c|c|c|c|c|}
\hline & \multicolumn{6}{|c|}{ Sufrieron violencia } \\
\hline & WV & & & $\mathrm{IIIX}$ & & \\
\hline & Frec. & $\%$ & Frec. & $\%$ & Frec. & $\%$ \\
\hline Tradicional & 68 & 28.9 & 29 & 12.3 & 97 & 41.2 \\
\hline Moderna & 98 & 41.7 & 40 & 17.0 & 138 & 58.7 \\
\hline TOTAL & 166 & 70.6 & 69 & 29.3 & 235 & 100.0 \\
\hline
\end{tabular}


lograr un fortalecimiento interno básico, para el fortalecimiento de su autoestima.

\section{BiblografíA}

1. Ortega MEC. Alteraciones psicológicas asociadas a la obesidad infantil. Revista Médica Herediana. 2018; 29 (2): $117-115$.

2. Lacunza A, Caballero S, Salazar R. Características de personalidad en adolescentes con sobrepeso y obesidad. Psicología y Salud. 2015; 25 (1): 5-14.

3. Kovalskys I, Rausch HC, Indart RP, Anez EV, Zonis LN, Orellana L. Childhood obesity and bullying in schools of Argentina: analysis of this behaviour in a context of high prevalence. Journal of childhood obesity. 2016; 1 (3): 1-9.

4. Herrera-López MR, Eva M, Ortega-Ruiz R. Bullying y Cyberbullying en Latinoamérica. Un estudio bibliométrico. Revista mexicana de investigación educativa. 2018; 23 (76): 125-155.

5. Montiel CMM, López RLF. Estilos parentales y su relación con la obesidad en niños de 2 a 8 años de edad. Revista mexicana de trastornos alimentarios. 2017 ; 8 (1): 11-20.

6. Caso NJ, Hernández-Guzmán L, González-Montesinos M. Prueba de Autoestima para Adolescentes. Universitas Psychologica. 2011; 10 (2): 535-543.

7. Orpinas P. Las escalas de agresióny victimización:validación y prevalencia en estudiantes. Revista Latinoamericana de Psicología. 2012; 44 (2): 109-124. 